\title{
EL PRESUPUESTO DE LOS ÓRGANOS GARANTES ESTATALES DEL DERECHO DE ACCESO A LA INFORMACIÓN EN MÉXICO, 2011-2017
} BUDGET OF SUBNATIONAL ENSURING BODIES OF ACCESS TO INFORMATION RIGHT IN MEXICO, 2011-2017

\section{José Said SÁNCHEZ MARTÍNEZ*}

RESUMEN. El objetivo de esta investigación es explorar si el partido en el gobierno y la composición partidista en el congreso inciden en los presupuestos de los órganos garantes del derecho de acceso a la información (OG). Para lograrlo, se ofrece información de los presupuestos durante el periodo 2011-2017, y mediante un análisis cuantitativo, se determina si las variables de interés tienen el efecto esperado. Los datos muestran que hay diferencias importantes en los presupuestos de los OG, y que bajo gobiernos divididos su presupuesto es mayor.

PALABRAS CLAVE. Órganos garantes subnacionales, presupuesto, gobierno unificado, gobierno dividido.

\begin{abstract}
This paper aims to explore if the political party in government and the partisan composition in congress have an effect on the budget of subnational bodies that ensure access to information right. The paper shows evidence about budgets in the period 2011-2017, and using a quantitative analysis, determines if the predictor variables have the expected effect. It concludes that there are differences between budgets, and that in a divided government scenario they are bigger.
\end{abstract}

* Profesor investigador de El Colegio de Jalisco; said.sanchez@coljal.edu.mx. Fecha de recepción: 25 de marzo de 2019.

Fecha de dictamen: 17 de junio de 2019. 
Esta revista forma parte del acervo de la Biblioteca Jurídica Virtual del Instituto de Investigaciones Jurídicas de la UNAM http://www.juridicas.unam.mx/ https://biblio.juridicas.unam.mx/bjv https://revistas.juridicas.unam.mx/

DOl: http://dx.doi.org/10.22201/iij.25940082e.2020.9.14279

JOSÉ SAID SÁNCHEZ MARTÍNEZ

KEY WORDS. Ensuring bodies, access to information, budget, divided government, unified government

\section{INTRODUCCIÓN}

86 El gasto público es la política más importante porque en él se determina cómo se distribuyen los recursos entre los diferentes temas y funciones del gobierno. Aunque parezca un asunto exclusivamente técnico, en su elaboración también se reflejan las preferencias de los participantes. En - términos formales, son el poder Ejecutivo y el Legislativo quienes se en-

- cargan de darle forma y contenido al presupuesto de egresos. Por ello, la pregunta de esta investigación es: ¿las variables partidistas inciden en los presupuestos de los órganos garantes?

Para responderla, este trabajo busca ofrecer evidencia sobre el presupuesto destinado a los órganos garantes (OG) durante el periodo 2011 2017. Los OG a los que refiere el trabajo están encargados de, entre otras funciones, hacer efectivo el derecho de acceso a la información gubernamental que tienen los ciudadanos (CIDE-COMAIP, 2014), por lo tanto, juegan un papel esencial en la política de transparencia y acceso a la información, así como en los esfuerzos por combatir a la corrupción.

Si bien algunos estudios han ofrecido datos sobre el presupuesto de los OG (CIDE-COMAIP, 2010, 2014; INOGDAI, 2014, 2016), no se ha hecho un análisis más detallado que muestre las tendencias y las diferencias entre los estados. Sin duda, un mayor gasto destinado a este rubro no implica en automático que habrá una mejora en la política de transparencia, pero se trata de un recurso indispensable para su funcionamiento.

$\mathrm{Al}$ respecto, dado "que los OG no pueden autorizar por sí mismos los recursos financieros que empleará, se considera que el presupuesto asignado a estos órganos es un indicador del interés y del compromiso de los gobiernos estatales y federal con la transparencia y el acceso a la información pública” (CIDE-COMAIP, 2014: 133). En términos sintéticos, el presupuesto es "un indicador del compromiso real del Estado con las diversas funciones y obligaciones de las autoridades públicas” (INOGDAI, 2016: 8).

A pesar de la reforma constitucional de 2014 en materia de transparencia, aún existen dudas sobre la suficiencia presupuestaria de los OG. Por ejemplo, el comisionado del Instituto Nacional de Transparencia, Acceso a la Información y Protección de Datos Personales (INAI), Óscar 
Guerra, expresó a finales de 2016 que los gobiernos estatales no se preocupan por la transparencia, ya que existen diferencias importantes en el gasto que destinan a este rubro. Para el comisionado, tal limitación impide cumplir al 100\% con las funciones de los OG (Morales, 2016). Incluso, los propios OG también han reconocido las limitaciones presupuestales para llevar a cabo sus funciones (Urrutia, 2016).

Sin duda, el escenario ideal sería que los recursos presupuestarios de los OG no estén al capricho de los cambios de gobierno y de las disputas partidistas entre el Ejecutivo y el Legislativo. No obstante, no debe olvidarse que a nivel subnacional se observa la convivencia de regímenes con diferentes características institucionales: algunos más próximos a la democracia y otros más similares al autoritarismo (Giraudy, 2015). Por ejemplo, se ha destacado que el margen de maniobra de los gobernadores "es mayor que el del presidente, porque... están menos vigilados y es mayor su capacidad de capturar congresos, tribunales, auditorías, órganos electorales y de acceso a la información locales" (Casar, 2017: 29). Paradójicamente, la "democracia dio a los gobernadores una autonomía notable, ya que en sus estados suelen controlar casi todo: desde el Congreso local hasta el Poder Judicial, pasando por los órganos supuestamente autónomos como los institutos electorales y las comisiones de derechos humanos" (Elizondo, 2012: 152). En suma, parece que los OG no se ubican en el mejor de los contextos políticos e institucionales para gozar de una mayor independencia presupuestaria.

Ya que los poderes Ejecutivo y Legislativo intervienen en la elaboración del presupuesto de los estados, las variables partidistas consideradas son la etiqueta o ideología del partido en el gobierno y la relación Ejecutivo-Legislativo (gobierno unificado o dividido). La hipótesis general es que estas variables inciden en los presupuestos de los OG. La información se obtuvo de las páginas web oficiales de los congresos, de los órganos públicos locales y de los presupuestos de egresos. El periodo de análisis (20112017) se determinó por cuestiones de disponibilidad de la información presupuestaria.

El artículo se estructura de la siguiente manera: en la primera sección se ofrece el argumento básico sobre los efectos que pueden tener la ideología del partido gobernante y la relación partidista Ejecutivo-Legislativo. Posteriormente, en la segunda sección se hace una descripción del gasto presupuestado de los OG. De forma comparativa, se muestran los datos respectivos de los órganos estatales de control interno (OECI) y de las entidades de fiscalización superior local (EFSL). Se hizo lo anterior porque 
junto con los OG, forman parte de los sistemas estatales anticorrupción de cada entidad y permiten tener una mejor idea de la magnitud del presupuesto de los OG. En esta sección también se realiza el análisis cuantitativo para determinar si las variables de interés están relacionadas con el presupuesto de los OG. Por último, se cierra con una sección de reflexiones.

- De manera general, el proceso presupuestario se compone de cuatro fases: - elaboración, aprobación, ejecución y control (Nacif, 2005). A nivel estatal, en términos esenciales, las Constituciones facultan a los gobernadores para diseñar y presentar el proyecto de presupuesto de egresos al congreso, el cual lo analiza, modifica y aprueba. Una vez aprobado, se devuelve al Ejecutivo para (si no hay observaciones) su publicación. Durante el año fiscal, el Ejecutivo se encarga de ejercer el gasto. Finalmente, las entidades de fiscalización superior locales verifican que los recursos se hayan erogado con apego a las leyes respectivas.

Debido al papel que juega el Poder Ejecutivo y el Poder Legislativo en la aprobación del gasto público, esta investigación busca no sólo ofrecer información sobre el presupuesto de los OG, sino también explorar si las variables partidistas inciden en su magnitud. Para ello, se consideran los argumentos del enfoque partidista en el aspecto de la ideología y en la relación Ejecutivo-Legislativo. En ambos argumentos los partidos políticos son esenciales para el tipo de decisiones que toman los gobiernos. Por un lado, los partidos políticos importan como fuente de ideología, la cual les permite no sólo distinguirse frente a los demás partidos, sino también reflejarla en el contenido de la política pública. Por el otro, los partidos importan como un instrumento de coordinación o conflicto entre el Poder Ejecutivo y el Poder Legislativo, dependiendo del tipo de relación partidista que exista entre ellos. A continuación se exponen ambos argumentos.

La teoría partidista argumenta que la ideología de los partidos políticos influye en el contenido y las características de las políticas públicas que serán implementadas una vez que estén en el gobierno (Hartmann, 2015; Schmitt, 2016). Aquí se entiende por ideología al sistema de ideas que permiten interpretar el mundo en diversos aspectos, desde el tipo de relaciones sociales hasta el papel que debe tener el Estado en la sociedad y la 
economía. La ideología de los partidos políticos se expresa en sus plataformas, manifiestos y programas (Vassallo y Wilcox, 2006), y permite no sólo identificar qué problemas merecen atención, sino también cómo se van a solucionar (Budge, 2006).

Los primeros argumentos que establecieron una correspondencia entre la ideología partidista y ciertas variables económicas fueron propuestos por Hibbs (1977). El autor parte de la idea de que el gobierno puede influir en los niveles de desempleo e inflación por medio de la manipulación de la política monetaria y fiscal. La causa de esta manipulación es que el gobierno responde a las preferencias de sus electores. Hibbs indica que los grupos sociales de ingresos bajos tienen más aversión por el desempleo que por la inflación, mientras que los de ingresos altos prefieren una inflación baja y un alto desempleo. Por lo tanto, las decisiones de política macroeconómica de los partidos en el gobierno reflejarán las preferencias de su electorado: los gobiernos de izquierda se enfocarán en reducir el desempleo y los gobiernos de derecha buscarán reducir la inflación.

La distinción entre un partido de derecha y uno de izquierda se basa en la postura que toman en cuestiones políticas relevantes, como son los aspectos económicos y sociales. Por ejemplo, se ha señalado que los partidos de izquierda tienen las siguientes preferencias (Hartmann, 2015): que los medios de producción estén en el sector público; un papel fuerte del gobierno en la planeación económica; apoyo a la distribución del ingreso desde los estratos altos hacia los bajos; expansión del estado de bienestar; bajo desempleo; alto gasto gubernamental; preferencia de la calidad sobre la cantidad del crecimiento económico, y bienestar para los menos privilegiados.

En contraste, las preferencias de los partidos de derecha son: que los medios de producción se ubiquen en el sector privado; un papel débil del estado en la planeación económica; oposición a la redistribución del ingreso; resistencia a la expansión del estado de bienestar; baja inflación; economía estable con un presupuesto balanceado y reducción del déficit; bajo gasto gubernamental; preferencia por el crecimiento económico per se, independientemente de sus efectos distributivos, y la no atención especial a los menos privilegiados.

Por otro lado, a partir de la revisión de diversos documentos de los partidos políticos y de la opinión de expertos, se ha demostrado que los gobiernos de derecha enfatizan la libertad, especialmente en la economía, buscan reducir el estado de bienestar, mantener la sociedad ordenada, 
y contar con una defensa militar fuerte, mientras que los partidos de izquierda prefieren expandir la esfera del gobierno, así como el estado de bienestar, la protección laboral y la paz internacional (Budge, 2006; Schmidt, 2010).

Igualmente, se ha demostrado que la distinción izquierda-derecha es una representación aceptable de las preferencias que tiene cada partido 90 político, y es entendida por las élites partidistas y por los electores (Budge, 2006; Vassallo y Wilcox, 2006). También se ha comprobado que las preferencias sobre determinadas políticas públicas están asociadas con la ubicación de los partidos en la dimensión izquierda-derecha (McDonald et al., 2007).

¿Qué muestra la evidencia sobre los efectos de la ideología partidista? Cabe destacar que la mayor parte de la investigación se ha basado en comparaciones internacionales ${ }^{1}$ y sus resultados son contrastantes: unos señalan que sí hay efectos, otros indican que son débiles o que dependen de otras condiciones (Herwartz y Theilen, 2014; Kittel y Obinger, 2003; Loftis y Mortensen, 2017; Potrafke, 2011; Russo y Verzichelli, 2016; Schmitt, 2016).

En México, sólo algunos estudios han considerado a la ideología partidista como variable explicativa en diversos temas del gasto público. Por ejemplo, Velázquez (2006) estudió el gasto total de los estados durante el periodo 1993-2002. Uno de sus hallazgos fue que los gobiernos panistas gastaron menos que los priistas, mientras que los perredistas gastaron más que los priistas.

Otro análisis es el de Ibarra (2013), quien se enfocó en la dependencia financiera de los estados durante el periodo 1998-2006. Sus hallazgos indican que hubo un efecto parcial de la ideología del partido que está en el gobierno.

Por su parte, Ingram (2012) estudió el gasto en el Poder Judicial en los estados durante el periodo 1993-2009. Entre sus resultados destaca que los gobiernos del Partido de la Revolución Democrática (PRD) y los del Partido Acción Nacional (PAN) gastaron más en sus poderes judiciales que los gobiernos del Partido Revolucionario Institucional (PRI).

En contraste con los resultados anteriores, la investigación de Montemayor (2003), la cual se enfocó en la deuda pública subnacional durante el periodo 1993-1999, no encontró un efecto del partido en el gobierno.

\footnotetext{
1 La mayoría de estos estudios analiza a los países integrantes de la Organización para la Cooperación y el Desarrollo Económicos en diferentes periodos.
} 
Respecto a la relación Ejecutivo-Legislativo, se ha argumentado que ella depende de dos elementos: los poderes constitucionales y los poderes partidistas del Ejecutivo (Mainwaring y Shugart, 2002). Los primeros consisten en los poderes formales inherentes al cargo del presidente. Mediante ellos puede cambiar o mantener el statu quo. Pero la efectividad de estos poderes depende de la dimensión partidista, es decir, del apoyo que tenga el Ejecutivo en el congreso. Como se ha señalado, uno "de los temas más importantes para las relaciones entre el Ejecutivo y el Legislativo de los sistemas presidenciales es el tamaño relativo del partido del presidente" (Mainwaring y Shugart, 2002: 257). Si el gobierno tiene la mayoría en el congreso, hay una unificación de propósitos; de lo contrario, emerge la separación. Lo anterior equivale a los términos más familiares de "gobierno unificado" y "gobierno dividido", respectivamente.

El modelo del gobierno de partido (party government) considera al partido político como un instrumento de unión que permite generar una acción coordinada y homogénea entre el Poder Ejecutivo y el Poder Legislativo, lo que podría generar un gobierno efectivo (Sundquist, 1988). Para que lo anterior ocurra, es necesario que el partido en el gobierno tenga la mayoría de escaños, que el presidente sea reconocido como el líder del partido y que existan altos grados de disciplina (Sundquist, 1988). Bajo tales condiciones, el presidente es quien determina las acciones del congreso y logra obtener apoyo para sus políticas. En otras palabras, los "frenos y contrapesos son neutralizados de forma considerable si el presidente y una mayoría disciplinada del mismo partido tienen un control unificado sobre el gobierno" (cursivas en original, Mainwaring y Shugart, 2002: 257).

Sin embargo, cuando ambas ramas se encuentran en manos de partidos diferentes, los efectos establecidos por el modelo del gobierno de partido son distintos. Bajo un gobierno dividido, el partido político del presidente tiene más obstáculos para coordinar la acción entre los poderes, ya que las preferencias partidistas son las que dominan su relación (Kernell, 1991). Por lo tanto, al sistema de pesos y contrapesos que existe en términos formales se añade la separación de propósitos (división partidista). Además, debido a la legitimidad democrática dual (Linz, 1998), derivada del hecho de que ambos poderes son electos mediante el voto popular, es más probable que bajo gobiernos divididos cada uno se proclame como el verdadero representante de la voluntad popular, lo que en ciertos casos podría generar parálisis política (Sundquist, 1988). No obstante, ante esta idea negativa también se ha señalado que el sistema de pesos y contrape- 
sos puede activar específicamente las funciones de control del congreso frente al Ejecutivo (Beer, 2001, 2000).

¿Qué indica la evidencia empírica? En las investigaciones realizadas para el congreso estadounidense aún no existe un consenso sobre el efecto del gobierno dividido en temas como la legislación (Binder, 2003, 1999; Coleman, 1999; Edwards, Barret y Peake, 1997; Howell et al., 2000; Kelly, 1993; Mayhew, 1991). En el tema del gasto público se encuentran diferencias más claras entre los gobiernos divididos y los unificados (McCubbins, 1991; Kiewiet y McCubbins, 1985a, 1985b).

En el caso mexicano, si bien en el nivel federal existe cierto consenso sobre el efecto del gobierno dividido, a nivel subnacional los resultados son contrastantes. En el caso del tema del gasto subnacional, se pueden destacar los siguientes estudios. Una de las primeras investigaciones fue la de Montemayor (2003), la cual buscó explicar los niveles de deuda de los gobiernos estatales entre 1993 y 1999. Halló que con gobierno unificado hubo mayor deuda que con gobierno dividido.

Carpizo (2012) también se enfocó en explicar los niveles de deuda pública a nivel subnacional, pero durante el periodo 2003-2010. Sus resultados señalan que los estados con gobiernos no unificados, específicamente con mayoría relativa, tuvieron más deuda que aquellos con gobiernos unificados.

La investigación de Armesto (2015) se enfocó en el gasto particularista en los gobiernos subnacionales de México y Argentina. Sus resultados indicaron que los gobiernos divididos no tienen un efecto sobre el gasto particularista.

Armesto y Olmeda (2018), por su parte, también analizaron el gasto particularista en los estados. Sus resultados mostraron que el gobierno dividido sí tuvo un impacto. Cabe señalar que este resultado es opuesto al de una publicación previa de los mismos autores (Armesto y Olmeda, 2016).

A partir de los argumentos expuestos, en esta investigación se establecen las siguientes hipótesis. En el caso del tema de la transparencia, ¿cómo afecta la ideología la postura del gobierno? Difícilmente algún gobernador va a expresarse en contra de ella en su plataforma de gobierno o en sus discursos, sobre todo ante la legitimidad que el tema ha ganado entre los ciudadanos y la academia. Por ejemplo, en el Plan Estatal de Desarrollo 2010-2016 del gobierno de César Duarte, quien ha sido acusado de diversos actos de corrupción, existe todo un programa a favor de la transparencia y la rendición de cuentas (Gobierno del Estado de Chihua- 
hua, 2010). Lo mismo ocurrió con el ex gobernador Javier Duarte, también señalado por actos de corrupción, en cuyo Plan Veracruzano de Desarrollo 2011-2017 se expresa: "Todos los actos y acciones de esta Administración habrán de caracterizarse por su transparencia y el comportamiento probo de los servidores públicos que participen" (Gobierno del Estado de Veracruz, 2011:34).

Si bien todos los partidos pueden expresarse a favor de la transparencia, lo que importa es el apoyo establecido en el presupuesto de los OG. De acuerdo con el argumento expuesto anteriormente, los partidos de derecha son más propensos a reducir el gasto público que los de izquierda. Por lo tanto, la hipótesis que se establece es:

H1: los estados gobernados por el PAN destinarán menos presupuesto a los OG en comparación con los otros gobiernos. ${ }^{2}$

En el caso de la relación Ejecutivo-Legislativo, ya que ambos poderes participan en la elaboración del presupuesto estatal, se argumenta que bajo gobiernos divididos, el congreso ejercerá su función de control frente al Ejecutivo. Uno de los instrumentos para ello es mediante el adecuado funcionamiento de los OG; por lo tanto, es posible que el congreso aumente su presupuesto. En el escenario opuesto, con gobierno unificado, el congreso respetará el contenido del presupuesto de egresos. En síntesis, la hipótesis es:

H2: bajo gobiernos divididos habrá un mayor presupuesto a los OG en comparación con los gobiernos unificados.

En la siguiente sección se describen los datos y los resultados del análisis estadístico.

2 Ya que durante el gobierno federal panista se creó la ley y el OG en materia de transparencia y acceso a la información pública, se podría plantear la hipótesis en la dirección opuesta, es decir, que los gobiernos estatales panistas estarán más comprometidos y destinarán más presupuesto a los OG. Sin embargo, la creación de estos organismos no implica per se un respaldo presupuestal en los siguientes años. Además, ya que el texto se concentra en cuestiones de gasto, se tomó en consideración cómo se comportarían los gobiernos en este aspecto de acuerdo con su ideología. 
Esta revista forma parte del acervo de la Biblioteca Jurídica Virtual del Instituto de Investigaciones Jurídicas de la UNAM

\section{El PRESUPUESTO DE LOS ÓRgANOS GARANTES, 2011-2017}

Como se indicó en la introducción, los OG son los encargados de hacer efectivo el derecho de acceso a la información gubernamental que tienen los ciudadanos. Pero también tienen otras facultades, como capacitar a los sujetos obligados, promover la cultura de la transparencia, expedir lineamientos y criterios de interpretación, vigilar que la información pública de oficio esté actualizada en los portales de los sujetos obligados, entre otras (CIDE-COMAIP, 2014). En consecuencia, tienen a su cargo diversas ta- reas sustantivas y forman una parte fundamental en la política de transpa-

- rencia y rendición de cuentas. En la tabla 1 se muestran las denominaciones actuales de los OG que se analizan en esta sección.

\section{Tabla 1. Órganos garantes en los estados}

\begin{tabular}{|l|l|}
\hline \multicolumn{1}{|c|}{ Estado } & \multicolumn{1}{c|}{ Órgano garante } \\
\hline Aguascalientes & Instituto de Transparencia del Estado de Aguascalientes \\
\hline Baja California & Instituto de Transparencia de Baja California \\
\hline $\begin{array}{l}\text { Baja California } \\
\text { Sur }\end{array}$ & $\begin{array}{l}\text { Instituto de Transparencia, Acceso a la Información y Protec- } \\
\text { ción de Datos Personales del Estado de Baja California Sur }\end{array}$ \\
\hline Campeche & $\begin{array}{l}\text { Comisión de Transparencia y Acceso a la Información Pública } \\
\text { del Estado de Campeche }\end{array}$ \\
\hline Coahuila & Instituto Coahuilense de Acceso a la Información Pública \\
\hline Colima & $\begin{array}{l}\text { Instituto de Transparencia, Acceso a la Información Pública y } \\
\text { Protección de Datos del Estado de Colima }\end{array}$ \\
\hline Chiapas & $\begin{array}{l}\text { Instituto de Acceso a la Información Pública del Estado de } \\
\text { Chiapas }\end{array}$ \\
\hline Chihuahua & $\begin{array}{l}\text { Instituto Chihuahuense para la Transparencia y Acceso a la } \\
\text { Información Pública }\end{array}$ \\
\hline Ciudad de & $\begin{array}{l}\text { Instituto de Transparencia, Acceso a la Información Pública, } \\
\text { Protección de Datos Personales y Rendición de Cuentas de la } \\
\text { Ciudad de México }\end{array}$ \\
\hline Durango & $\begin{array}{l}\text { Instituto Duranguense de Acceso a la Información Pública y } \\
\text { de Protección de Datos Personales }\end{array}$ \\
\hline Guanajuato & $\begin{array}{l}\text { Instituto de Acceso a la Información Pública para el Estado de } \\
\text { Guanajuato }\end{array}$ \\
\hline Guerrero & $\begin{array}{l}\text { Instituto de Transparencia, Acceso a la Información y Protec- } \\
\text { ción de Datos Personales del Estado de Guerrero }\end{array}$ \\
\hline
\end{tabular}


Esta revista forma parte del acervo de la Biblioteca Jurídica Virtual del Instituto de Investigaciones Jurídicas de la UNAM

\begin{tabular}{|c|c|}
\hline Estado & Órgano garante \\
\hline Hidalgo & $\begin{array}{l}\text { Instituto de Transparencia, Acceso a la Información Pública } \\
\text { Gubernamental y Protección de Datos Personales del Estado } \\
\text { de Hidalgo }\end{array}$ \\
\hline Jalisco & $\begin{array}{l}\text { Instituto de Transparencia, Información Pública y Protección } \\
\text { de Datos Personales del Estado de Jalisco }\end{array}$ \\
\hline $\begin{array}{l}\text { Estado de } \\
\text { México }\end{array}$ & $\begin{array}{l}\text { Instituto de Transparencia, Acceso a la Información Pública y } \\
\text { Protección de Datos Personales del Estado de México y Mu- } \\
\text { nicipio }\end{array}$ \\
\hline Michoacán & $\begin{array}{l}\text { Instituto Michoacano de Transparencia, Acceso a la Informa- } \\
\text { ción y Protección de Datos Personales }\end{array}$ \\
\hline Morelos & Instituto Morelense de Información Pública y Estadística \\
\hline Nayarit & $\begin{array}{l}\text { Instituto de Transparencia y Acceso a la Información Pública } \\
\text { del Estado de Nayarit }\end{array}$ \\
\hline Nuevo León & $\begin{array}{l}\text { Comisión de Transparencia y Acceso a la Información del Es- } \\
\text { tado de Nuevo León }\end{array}$ \\
\hline Oaxaca & $\begin{array}{l}\text { Instituto de Acceso a la Información Pública y Protección de } \\
\text { Datos Personales del Estado de Oaxaca }\end{array}$ \\
\hline Puebla & $\begin{array}{l}\text { Instituto de Transparencia, Acceso a la Información Pública y } \\
\text { Protección de Datos Personales del Estado de Puebla }\end{array}$ \\
\hline Querétaro & $\begin{array}{l}\text { Comisión de Transparencia y Acceso a la Información Pública } \\
\text { del Estado de Querétaro }\end{array}$ \\
\hline Quintana Roo & $\begin{array}{l}\text { Instituto de Acceso a la Información y Protección de Datos } \\
\text { Personales de Quintana Roo }\end{array}$ \\
\hline $\begin{array}{l}\text { San Luis } \\
\text { Potosí }\end{array}$ & $\begin{array}{l}\text { Comisión Estatal de Garantía de Acceso a la Información Pú- } \\
\text { blica del Estado de San Luis Potosí }\end{array}$ \\
\hline Sinaloa & Comisión Estatal para el Acceso a la Información Pública \\
\hline Sonora & $\begin{array}{l}\text { Instituto Sonorense de Transparencia, Acceso a la Informa- } \\
\text { ción Pública y Protección de Datos Personales }\end{array}$ \\
\hline Tabasco & $\begin{array}{l}\text { Instituto Tabasqueño de Transparencia y Acceso a la Informa- } \\
\text { ción Pública }\end{array}$ \\
\hline Tamaulipas & $\begin{array}{l}\text { Instituto de Transparencia y Acceso a la Información de Ta- } \\
\text { maulipas }\end{array}$ \\
\hline Tlaxcala & $\begin{array}{l}\text { Instituto de Acceso a la Información Pública y Protección de } \\
\text { Datos Personales del Estado de Tlaxcala }\end{array}$ \\
\hline Veracruz & $\begin{array}{l}\text { Instituto Veracruzano de Acceso a la Información y Protección } \\
\text { de Datos Personales }\end{array}$ \\
\hline Yucatán & $\begin{array}{l}\text { Instituto Estatal de Transparencia, Acceso a la Información } \\
\text { Pública y Protección de Datos Personales }\end{array}$ \\
\hline
\end{tabular}


Esta revista forma parte del acervo de la Biblioteca Jurídica Virtual del Instituto de Investigaciones Jurídicas de la UNAM http://www.juridicas.unam.mx/

\begin{tabular}{|c|l|}
\hline Estado & \multicolumn{1}{c|}{ Órgano garante } \\
\hline Zacatecas & $\begin{array}{l}\text { Instituto Zacatecano de Transparencia y Acceso a la Informa- } \\
\text { ción y Protección de Datos Personales }\end{array}$ \\
\hline
\end{tabular}

Fuente: elaboración propia con información de las páginas oficiales de los OG. Fecha de consulta: 26 de junio de 2019.

Se ha señalado que todos los OG del país cuentan con una autonomía reconocida, que existe una mejora en la creación de manuales de operación y procedimientos, que han aumentado sus atribuciones y universos de acción, y que los comisionados están en condiciones de ejercer su mandato de tiempo completo, entre otros avances. No obstante, también se ha destacado que los OG tienen retos importantes. Por ejemplo, no se ha desarrollado un servicio profesional de carrera efectivo, hay un estancamiento en la asignación presupuestal y cuentan con un poder débil de incidencia en los sujetos obligados (CIDE-COMAIP, 2014).

En relación con el presupuesto de los OG, el CIDE-COMAIP (2014) analizó información en el periodo 2010-2013. Uno de los resultados fue que el presupuesto asignado a los OG no fue tan significativo como en los primeros años de institucionalización de tales organismos (CIDECOMAIP, 2014). Como se indicó en la introducción, si bien un presupuesto suficiente no garantiza un buen desempeño, sin duda es esencial para las tareas de los OG. Además, a pesar de la reciente reforma constitucional en materia de transparencia, aún se muestran preocupaciones por la suficiencia de recursos de los OG.

¿Cuál es la situación actual? En la gráfica 1 se muestra el presupuesto promedio anual de los OG para las 32 entidades durante el periodo 2011 2017. Para tener una mejor compresión de las cifras, de manera comparada se considera el presupuesto de los OECI y de las EFSL. ${ }^{3}$ Además, los tres organismos forman parte de los sistemas estatales anticorrupción de cada entidad, por lo que resulta pertinente la comparación. Destaca que el presupuesto de los OG es mucho menor que el de las otras dos instituciones. En 2011 los OG registraron un presupuesto promedio de 21.8 millones de pesos y cerraron 2017 con 26.4 millones (a precios de 2013). Por su parte, el presupuesto de los OECI ha oscilado entre 83.7 y 89.5 millones de pesos, mientras que el de las EFSL ha oscilado entre 84.9 y 109.7 millones de pesos. A lo largo del periodo, los OG han registrado un aumento discreto pero constante, lo cual también ocurrió en los OECI y las EFSL.

\footnotetext{
3 En el caso de los estados de Baja California Sur, Michoacán y Guanajuato no se pudo obtener la información correspondiente para sus EFSL.
} 
Esta revista forma parte del acervo de la Biblioteca Jurídica Virtual del Instituto de Investigaciones Jurídicas de la UNAM

Gráfica 1. Presupuesto promedio anual de los OG, 2011-2017

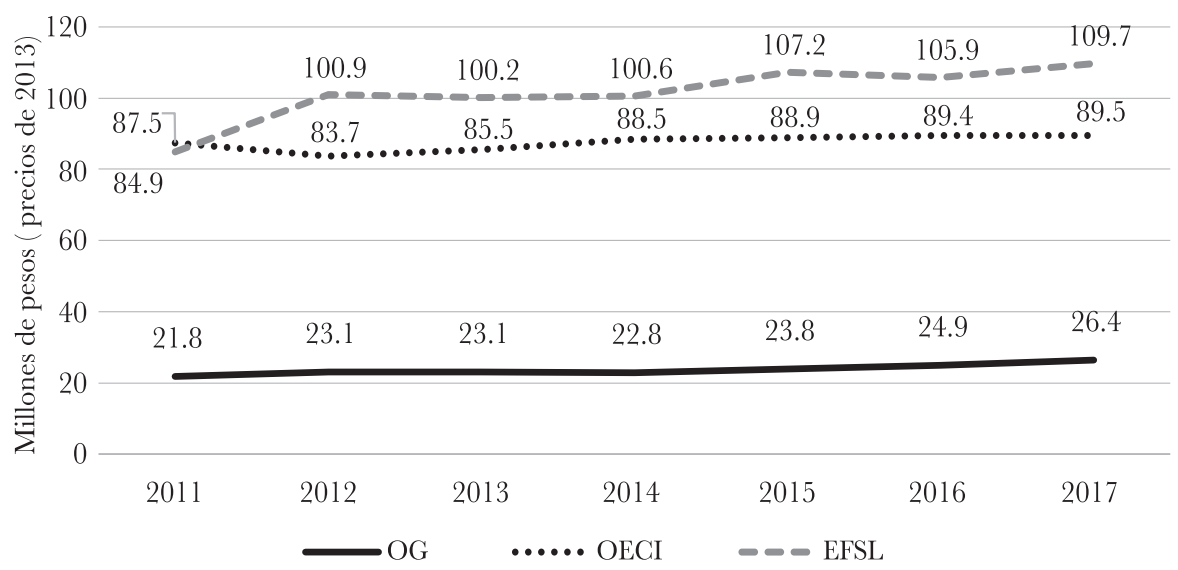

FuENTE: elaboración propia con datos de los presupuestos de egresos de cada estado.

Sin embargo, los datos anteriores ocultan las diferencias entre los estados. En la gráfica 2 se expone el presupuesto promedio por estado durante el periodo 2011-2017. El promedio general del gasto presupuestado de los OG es de 23.7 millones de pesos. Se aprecia de manera clara que el OG de la Ciudad de México se aleja considerablemente del promedio general, con un presupuesto promedio de 113.8 millones de pesos. Le sigue el OG del Estado de México, con 77 millones, y en tercer lugar, Jalisco, con 44.1 millones de pesos. En el extremo opuesto se ubica Baja California Sur, con un presupuesto promedio de 5.7 millones de pesos, seguido de Colima y Nayarit con 5.9 y 6 millones de pesos, respectivamente.

Los datos también muestran de manera clara que los presupuestos de los OG están muy por debajo de los correspondientes a los OECI y las EFSL. No obstante, en algunos casos las diferencias son más amplias. Por ejemplo, en Chiapas el presupuesto promedio del OG fue de 11.4 millones de pesos, mientras que el OECI y la EFSL tuvieron 171.5 y 125.8 millones respectivamente, es decir, más de diez veces el monto de aquél. Una situación similar se aprecia en Puebla, cuyo OG tuvo un presupuesto promedio de 12.2 millones, mientras que el OECI y la EFSL tuvieron 140.7 y 127.6 millones de pesos respectivamente. En otros estados la diferencia ha sido menor. En Aguascalientes el presupuesto promedio del OG, OECI y EFSL ha sido de 12.3, 29.7 y 21.3 millones de pesos respectivamente. Asimismo, en Morelos el OG tuvo un presupuesto de 16 millones, el OECI contó con 14 millones y la EFSL con 6.2 millones de pesos. 
Esta revista forma parte del acervo de la Biblioteca Jurídica Virtual del Instituto de Investigaciones Jurídicas de la UNAM http://www.juridicas.unam.mx/ https://biblio.juridicas.unam.mx/bjv

\section{Gráfica 2. Presupuesto promedio de los OG POR ESTADO, 2011-2017}

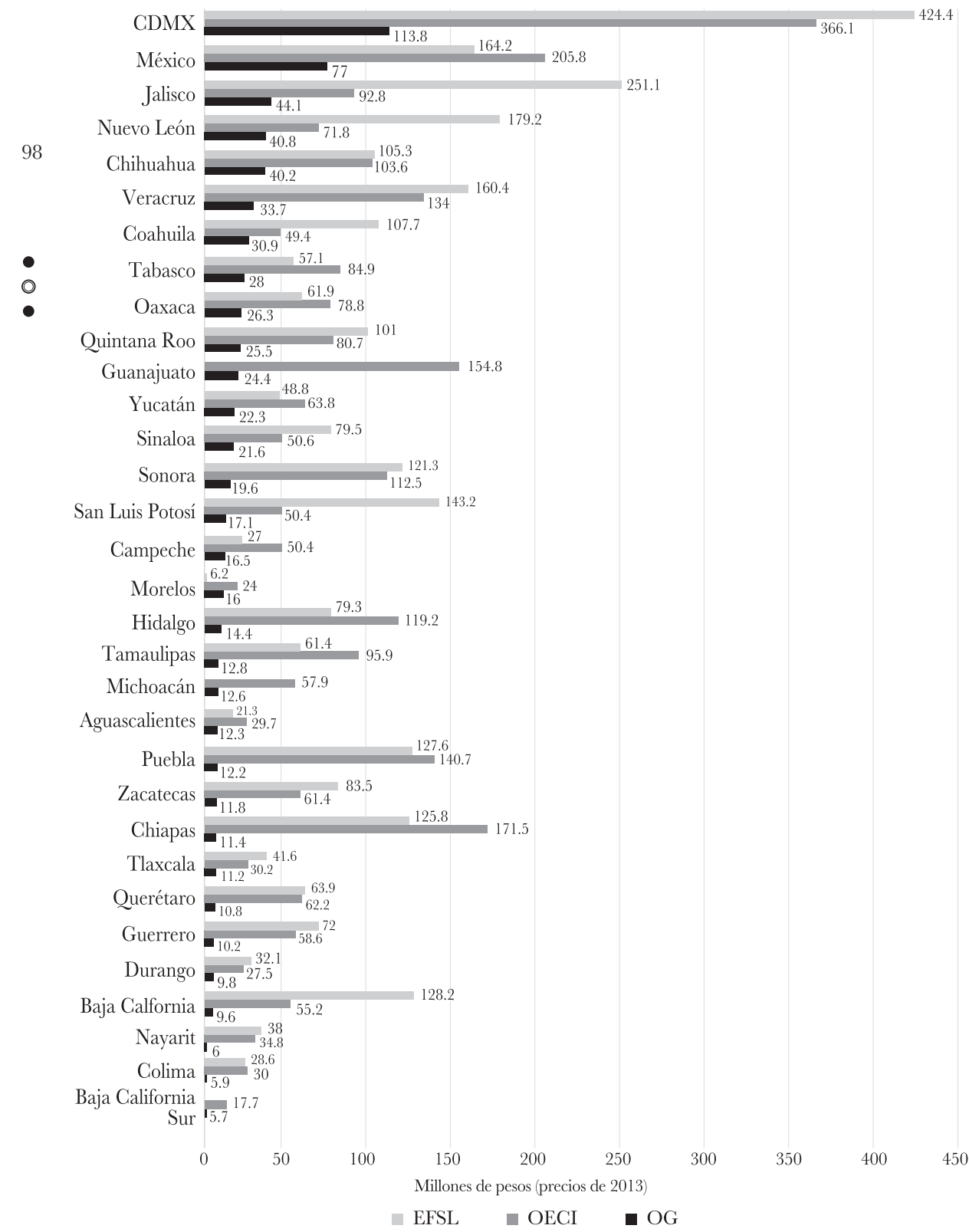

FueNTE: elaboración propia con datos de los presupuestos de egresos de cada estado. 
Lo anterior es sólo un primer acercamiento. No obstante, es preciso controlar el presupuesto por la población de cada estado. En tal sentido, en la gráfica 3 se expone el presupuesto per cápita de los OG, OECI y EFSL. Con este indicador, el promedio presupuestal de los OG es de 7.2 pesos por habitante. El OG de Campeche es el que tiene la mayor cantidad, con 18.3 pesos por habitante, seguido de Quintana Roo, con 17.2, y la Ciudad de México, con 12.6 pesos. En el lado opuesto se encuentra el OG de Puebla, con sólo 2 pesos por habitante. Le siguen Chiapas y Michoacán con 2.2 y 2.7 pesos.

Las diferencias se mantienen incluso cuando se comparan a estados con una población similar. Por ejemplo, Chihuahua y Tamaulipas cuentan con 3.8 y 3.6 millones de habitantes respectivamente; sin embargo, en el primer estado el OG tiene un presupuesto promedio de 11.2 pesos por habitante, y el segundo, uno de 3.7 pesos. Otro par de casos son Coahuila y Guerrero, con una población de 3 y 3.6 millones respectivamente, pero el $\mathrm{OG}$ del primero tiene un presupuesto promedio de 10.4 pesos, y el del segundo, uno de 2.9 pesos por persona.

Con este indicador también se mantienen las diferencias entre los presupuestos de las tres instituciones. Las diferencias extremas se ubican en Puebla y Chiapas. En Puebla, el presupuesto del OECI y de la EFSL supera en poco más de diez veces al del OG, ya que contaron con 22.8 y 20.6 pesos por habitante, mientras que aquél tuvo un promedio de 2 pesos. En Chiapas, el OG registró un gasto promedio de 2.2 pesos, en tanto que el OECI y la EFSL tuvieron 33 y 20.6 pesos por habitante. Algunos de los estados con los menores contrastes son Campeche, Tabasco, Yucatán, Coahuila, Aguascalientes, Oaxaca y Durango, en donde los OECI y las EFSL tuvieron un presupuesto que duplicó o triplicó al de los OG. 
Esta revista forma parte del acervo de la Biblioteca Jurídica Virtual del Instituto de Investigaciones Jurídicas de la UNAM http://www.juridicas.unam.mx/

\section{Gráfica 3. Presupuesto promedio de los OG, 2011-2017}

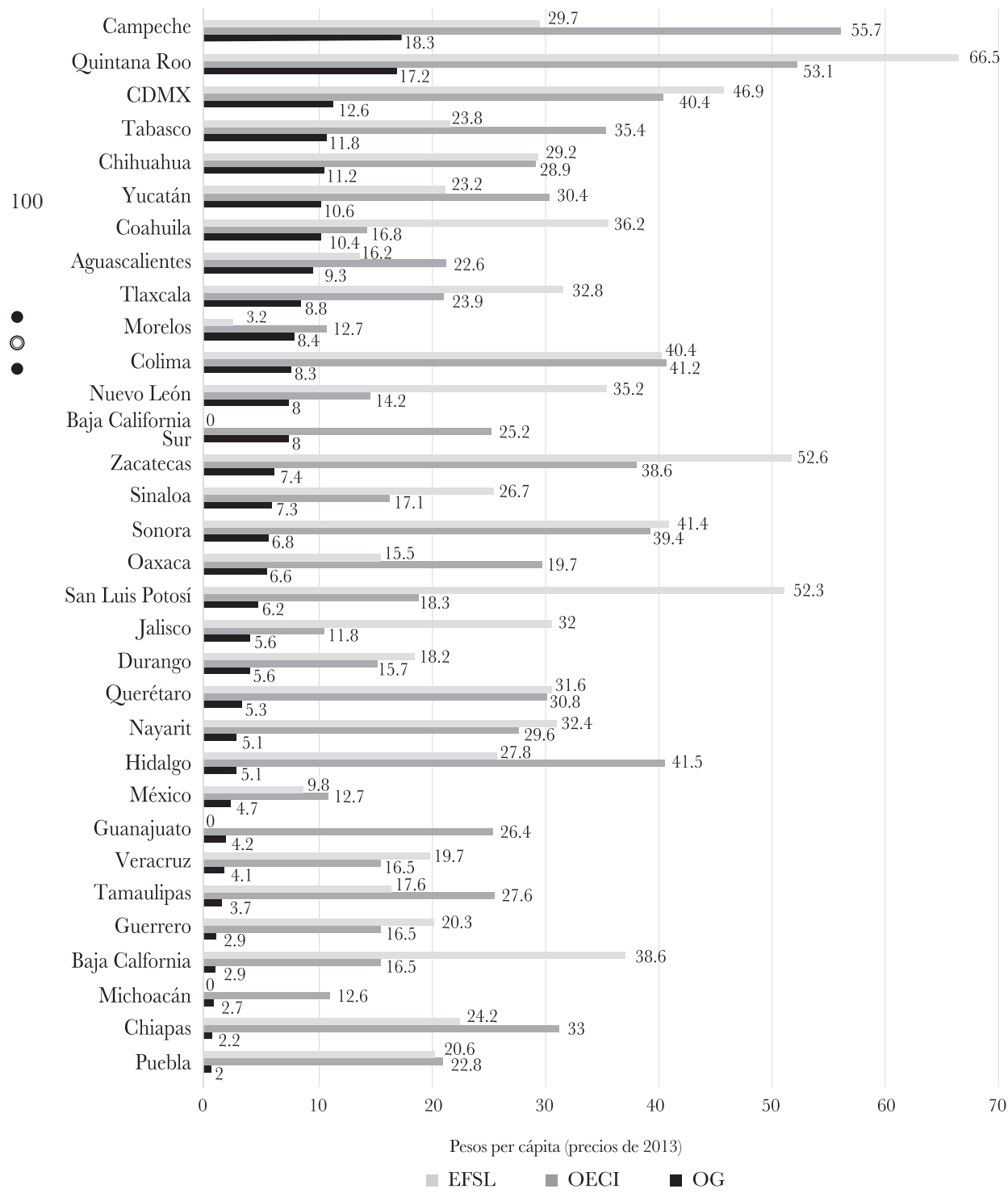

Fuente: elaboración propia con datos de los presupuestos de egresos de cada estado e INEGI.

Finalmente, hay que observar cómo se ha comportado el presupuesto de los OG a lo largo del tiempo en cada estado. Por cuestiones de claridad, en la gráfica 4 sólo se muestra el presupuesto de los OG. La variación en 
Esta revista forma parte del acervo de la Biblioteca Jurídica Virtual del Instituto de Investigaciones Jurídicas de la UNAM

el presupuesto al interior de cada estado ha sido poca. No obstante, se pueden destacar algunos estados con cambios que no hay que pasar por alto. Por ejemplo, en Aguascalientes, entre 2011 y 2015 el OG tuvo un presupuesto entre los 7 y 8 pesos por habitante, pero subió en 2016 y 2017 a 13.9 y 12.9 pesos, respectivamente. En Nayarit se observa que en 2011 el presupuesto del OG fue de 3.7 pesos por habitante, en 2014 llegó a 4.9 pesos y en 2017 cerró con 7.9. También se puede mencionar a Zacatecas, cuyo OG tuvo un presupuesto en 2011 de 5.8 pesos, en 2015 subió a 7.4, y cerró 2017 con 10.4 pesos por habitante. Por el otro lado, hay que señalar a los estados que han mantenido constante el nivel de presupuesto de sus OG. Por ejemplo, Colima ha mantenido un presupuesto entre 7.6 y 8.9 pesos por habitante; en Tamaulipas ha oscilado entre 3.5 y 3.9 pesos, y en Puebla, entre 1.8 y 2.1 pesos por habitante. Mención aparte merece Quintana Roo, en donde al inicio del periodo registró un presupuesto de 18.6 pesos por habitante, pero se fue reduciendo hasta los 14.4 pesos en 2015, y volvió a aumentar a 22.6 pesos en 2017.

\section{Gráfica 4. Presupuesto per cápita de los OG POR ESTADO, 2011-2017}

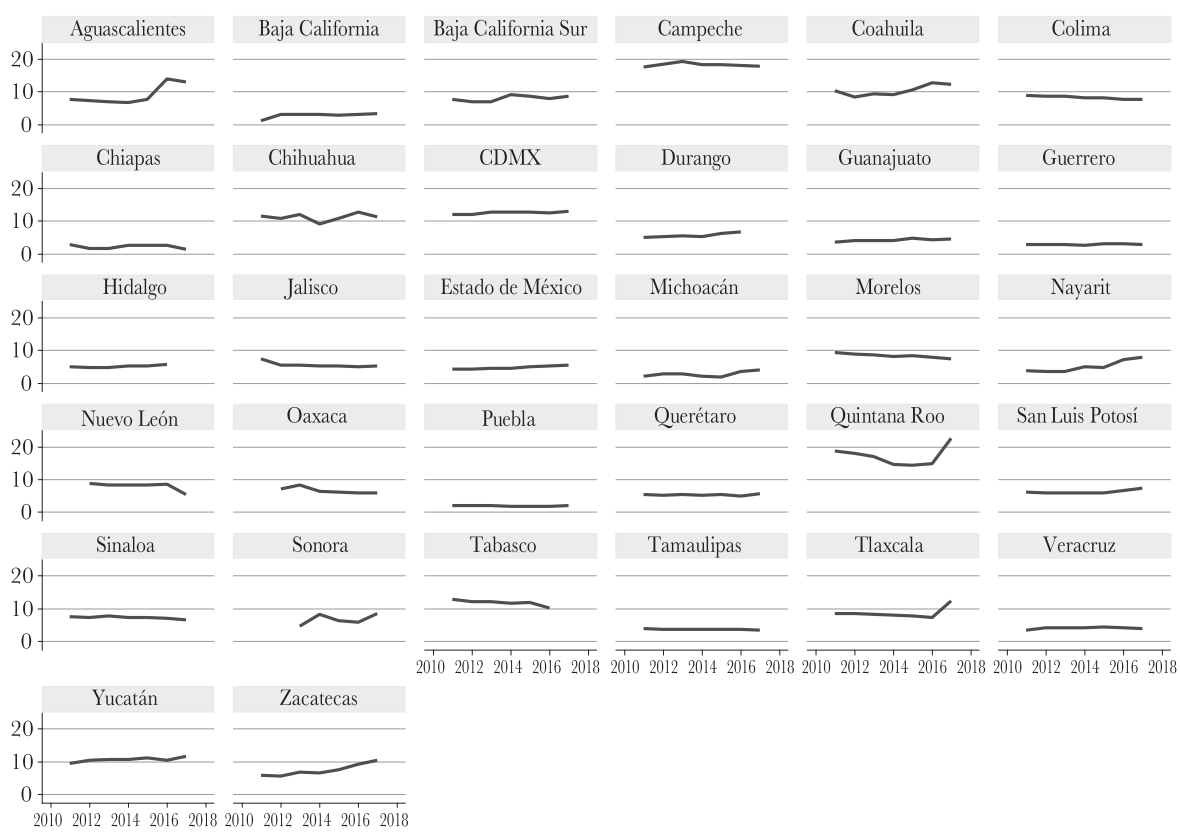

Fuente: elaboración propia con datos de los presupuestos de egresos de cada estado e INEGI. 
Esta revista forma parte del acervo de la Biblioteca Jurídica Virtual del Instituto de Investigaciones Jurídicas de la UNAM

Los datos anteriores muestran que hay importantes diferencias en los presupuestos de los OG de cada estado. Incluso éstas persisten entre estados con poblaciones similares. Si el presupuesto destinado a los OG se puede interpretar como un compromiso del gobierno con el tema de la transparencia y el acceso a la información, ¿por qué unos estados muestran más compromiso que otros?, ¿qué variables podrían explicar las diferencias presupuestales? Ya que el poder Ejecutivo y Legislativo se encargan de diseñar y aprobar el presupuesto del estado, es preciso determinar si el presupuesto de los OG es afectado por el partido político en el gobierno y por el tipo de relación partidista de éste con el congreso. Para comprobar - lo anterior, se hará un análisis para datos de tipo panel. El panel cuenta - con datos para las 32 entidades durante el periodo 2011-2017. En la tabla 2 se expone la estadística descriptiva de las variables de interés. Se observa que durante el periodo de estudio el $12 \%$ de los estados fueron gobernados por el PAN, $62 \%$ por el PRI, $27 \%$ por el PRD, y $11 \%$ por una alianza PANPRD. En el caso de la relación Ejecutivo-Legislativo, el 64\% de los estados estuvo en un contexto de gobiernos divididos, mientras que el resto tuvo gobiernos unificados.

TABLA 2. Estadística descriptiva

\begin{tabular}{|l|c|c|c|c|c|}
\hline \multicolumn{1}{|c|}{ Variable } & Media & $\begin{array}{c}\text { Des- } \\
\text { viación } \\
\text { estándar }\end{array}$ & Mínimo & Máximo & $\begin{array}{c}\text { Obser- } \\
\text { vacio- } \\
\text { nes }\end{array}$ \\
\hline $\begin{array}{l}\text { Presupuesto per cápi- } \\
\text { ta de los OG }\end{array}$ & 7.2 & 4.1 & 1.3 & 22.6 & 213 \\
\hline $\begin{array}{l}\text { Presupuesto (precios } \\
\text { de 2013) }\end{array}$ & 23.6 & 22.4 & 4.1 & 116.6 & 213 \\
\hline Partido en el gobierno & 1.2 & 0.8 & 0 & 3 & 222 \\
\hline PAN & & & & & $\begin{array}{c}32 \\
(14 \%)\end{array}$ \\
\hline PRI ${ }^{2}$ & & & & & $\begin{array}{c}138 \\
(62 \%)\end{array}$ \\
\hline PRD & & & & & 27 \\
\hline PAN-PRD & 0.6 & 0.5 & 0 & 1 & $22 \%$ \\
\hline Estatus del gobierno & & & & & 143 \\
\hline Gobierno dividido & & & & & $(64 \%)$ \\
\hline
\end{tabular}


Esta revista forma parte del acervo de la Biblioteca Jurídica Virtual del Instituto de Investigaciones Jurídicas de la UNAM

\begin{tabular}{|l|l|l|l|l|c|}
\hline Gobierno unificado & & & & $\begin{array}{c}79 \\
(36 \%)\end{array}$ \\
\hline $\begin{array}{l}\text { PIB estatal (miles de } \\
\text { millones) }\end{array}$ & $497,571.8$ & $513,592.2$ & $86,031.7$ & $3,044,809$ & 222 \\
\hline
\end{tabular}

FuENTE: elaboración propia con datos del INEGI, los presupuestos de egresos de cada estado, los organismos públicos locales electorales y solicitudes de acceso a la información a los congresos estatales.

1 Pesos a precios de 2013.

2 Ya que el PVEM gobernó un solo estado y ganó en alianza con el PRI (Chiapas), se contabilizó en el grupo de este partido.

3 INEGI, precios de 2013.

Después de aplicar las pruebas correspondientes, se determinó utilizar efectos fijos. Ya que se detectaron problemas de heteroscedasticidad y autocorrelación, se utilizaron estimadores con errores estándar corregidos para panel (panel corrected standard errors o PCSE). El modelo es:

$$
P O G_{i, t}=\beta 1 p g_{i, t}+\beta 2 g d_{i, t}+\beta 4 l p i b_{i, t}+E_{i}+\varepsilon_{i, t}
$$

En donde POG es el presupuesto per cápita del OG; pg es la variable categórica para el partido político en el gobierno $(\mathrm{PAN}$ : pgl $1=0, \mathrm{pg} 2=0$, pg3=0; PRI: pgl=1, pg2=0, pg3=0; PRD: pgl=0, pg2=1, pg3=0; PANPRD: $p g 1=0, p g 2=0, p g 3=1)$; gd es una variable dicotómica para la relación partidista Ejecutivo-Legislativo (gobierno dividido vertical=1; gobierno unificado vertical=0); lpib es el logaritmo natural del producto interno bruto estatal, y $E$ se refiere a las características de los estados. Además, se añade otro modelo considerando el presupuesto bruto en millones de pesos (a precios de 2013), en el cual se controla por el PIB y la población.

Los resultados se muestran en la tabla 3. Se observa que, tomando como grupo de referencia a los gobiernos del PAN, contrario a la dirección de la hipótesis H1, los gobiernos del PRI y del PRD destinan menos presupuesto a sus respectivos OG. En promedio, destinan 0.6 y 0.7 pesos per cápita menos comparados con el PAN. En el caso de los gobiernos del PAN-PRD, se obtiene un signo positivo, pero el resultado no es estadísticamente significativo. No se puede confirmar la dirección de la H1. En el caso de los gobiernos divididos, se aprecia que en estas circunstancias los

4 Cabe señalar que no se incluyeron los años correspondientes del gobierno de Jaime Rodríguez Calderón, ya que al ser el único sin una afiliación partidista tiene pocas observaciones. 
Esta revista forma parte del acervo de la Biblioteca Jurídica Virtual del Instituto de Investigaciones Jurídicas de la UNAM

OG tienen 0.5 pesos per cápita más comparados con los gobiernos unificados, lo cual permite confirmar la hipótesis H2.

Sin embargo, los resultados del segundo modelo muestran algunas diferencias. En primer lugar, con respecto a los efectos de la ideología de los partidos gobernantes, se observa que los signos son los mismos del modelo 1, pero en este caso dejan de ser estadísticamente significativos. En otros términos, no se puede afirmar que hay diferencias en los presupuestos de los OG en función del partido en el gobierno. En el caso de la relación partidista Ejecutivo-Legislativo, se aprecia que nuevamente se confirma el signo esperado, el cual es estadísticamente significativo. En este contexto, el presupuesto de los OG es, en promedio, 1.4 millones mayor comparado con escenarios de gobierno unificado.

\section{Tabla 3. Resultados}

\begin{tabular}{|c|c|c|}
\hline Variable & $\begin{array}{l}\text { Modelo } 1 \text {. } \\
\text { Presupuesto per cápita }\end{array}$ & $\begin{array}{c}\text { Modelo } 2 . \\
\text { Presupuesto (millones de } \\
\text { pesos a precios de 2013) }\end{array}$ \\
\hline PRI & $\begin{array}{l}-0.6459^{*} \\
(0.3809)\end{array}$ & $\begin{array}{l}-1.4944 \\
(1.1061)\end{array}$ \\
\hline PRD & $\begin{array}{c}-0.7098^{* *} \\
(0.3813)\end{array}$ & $\begin{array}{l}-0.8770 \\
(1.1803)\end{array}$ \\
\hline PAN-PRD & $\begin{array}{c}0.6576 \\
(0.4400)\end{array}$ & $\begin{array}{l}1.0741 \\
(1.1114)\end{array}$ \\
\hline Gobierno dividido & $\begin{array}{l}0.5072^{* *} \\
(0.2275)\end{array}$ & $\begin{array}{l}1.4421^{* *} \\
(0.6005)\end{array}$ \\
\hline $\begin{array}{l}\text { PIB estatal (logaritmo } \\
\text { natural) }\end{array}$ & $\begin{array}{c}4.7944^{* * *} \\
(1.2219)\end{array}$ & $\begin{array}{l}13.0421^{* * *} \\
(3.7062)\end{array}$ \\
\hline $\begin{array}{l}\text { Población (logaritmo na- } \\
\text { tural) }\end{array}$ & & $\begin{array}{l}14.4103^{*} \\
(8.0046)\end{array}$ \\
\hline $\mathrm{R}^{2}$ & 0.9329 & 0.9808 \\
\hline Observaciones & 213 & 213 \\
\hline $\begin{array}{rl}* & p<=0.1 \\
* * & p \\
* * * & =0.05 \\
\mathrm{p} & <=0.01\end{array}$ & & \\
\hline
\end{tabular}




\section{REFLEXIONes FinALES}

Los datos sobre el presupuesto destinado a los OG muestran que existen diferencias importantes entre los estados. Incluso, las diferencias persisten una vez que se controla por la población. Si se considera que los OG tienen funciones relativamente similares, entonces existe un serio problema, ya que las están ejerciendo con recursos presupuestarios desiguales. Sin duda, un presupuesto suficiente no implica que en automático estos órganos se desempeñarán de manera eficiente y eficaz. No obstante, se trata de un elemento necesario para su funcionamiento. De forma comparativa, se mostraron los presupuestos respectivos de los OECI y las EFSL para dar cuenta de la magnitud de los recursos de los OG. En términos generales, se observó que los OG cuentan con un presupuesto mucho menor.

Si se considera el presupuesto de los OG como un indicador del compromiso de los gobiernos con el tema de la transparencia y el acceso a la información, entonces se puede decir que no todos tienen el mismo nivel de compromiso. ¿Qué podría explicar lo anterior? De forma exploratoria, aquí se buscó determinar si el partido en el gobierno y la relación partidista Ejecutivo-Legislativo están relacionados con el nivel de presupuesto dirigido a los OG. En el caso de la ideología del partido en el gobierno, los resultados mostraron un signo contrario al esperado, es decir, los gobiernos panistas destinaron más presupuesto a los OG. Además, la significancia estadística no se mantuvo en los dos modelos propuestos. Por ello, con la información disponible no se puede afirmar que la ideología partidista tenga un efecto sobre los presupuestos de los OG. En futuros trabajos será preciso ampliar el periodo de estudio para obtener más observaciones y resultados más robustos. Lamentablemente, lo anterior a menudo depende de la disponibilidad de la información presupuestal.

En el caso del gobierno dividido, los resultados mostraron que en este contexto los OG tuvieron un mayor presupuesto que bajo gobiernos unificados. Lo anterior se confirmó en los dos modelos. Esto podría significar que cuando el gobernador no tiene la mayoría en el congreso, la oposición trata de que los OG tengan los recursos necesarios para su funcionamiento. En otras palabras, con gobiernos divididos el congreso muestra un mayor compromiso con la política de transparencia y acceso a la información en comparación con el escenario opuesto. En futuras investigaciones será necesario mostrar el mecanismo causal que conecta ambas variables.

En síntesis, los datos muestran que hay variaciones considerables en los presupuestos de los OG. A pesar de la reciente reforma constitucional 
en materia de transparencia y acceso a la información, las diferencias presupuestales persisten. Será preciso hacer un análisis temporal más amplio y considerar otras variables explicativas. Finalmente, el siguiente paso en la investigación es identificar los efectos concretos que tiene el presupuesto sobre el desempeño de los OG y sobre el combate a la corrupción.

Armesto, María Alejandra, 2015, "Competencia política y gasto particularista de los gobiernos subnacionales en México y Argentina”, Latin American Research Review, vol. 50, núm. 1.

Armesto, María Alejandra y Olmeda Juan C., 2018, "Oposición legislativa y patronazgo político. Gasto en empleo público de los estados en México, 2001-2012”, Región y Sociedad, vol. 30, núm. 71.

Armesto, María Alejandra y Olmeda Juan C., 2016, "Estrategias de interacción Ejecutivo-Legislativo y patronazgo político subnacional en México”, en LozA, Nicolás y Méndez, Irma, (coords.), Poderes y democracias. La política subnacional en México, Ciudad de México, Flacso-IEDF.

Beer, Caroline, 2001, "Assessing the Consequences of Electoral Democracy: Subnational Legislative Change in Mexico", Comparative Politics, vol. 33, núm. 4.

BeEr, Caroline, 2000, Assessing the Consequences of Electoral Competition: Legislative Reform, Political Recruitment, and Fiscal Policy in the Mexican States, tesis doctoral inédita, Albuquerque, The University of New Mexico.

Binder, Sarah A., 2003, Stalemate. Causes and Consequences of Legislative Gridlock, Virginia, Brookings Institution Press.

Binder, Sarah A., 1999, "The Dynamics of Congressional Gridlock, $1947-$ 1996", American Political Science Review, vol. 93, núm. 3.

Budge, Ian, 2006, "Identifying Dimensions and Locating Parties: Methodological and Conceptual Problems", en Katz, Richard у Свотту, William J. (eds.), Handbook of Party Politics, Londres, Sage.

Carpizo, Carlos I., 2012, Los efectos cruzados de la competencia política sobre el endeudamiento: un análisis empírico de la deuda estatal en México, 2003-2010, trabajo presentado en el marco del Premio Nacional de Finanzas Públicas 2012, Ciudad de México, Cámara de Diputados. 
Casar, María Amparo, 2017, "La sombra de las instituciones", en AguiLAR, Héctor et al. (coords.), ¿Y ahora qué? México ante el 2018, México, Debate.

Centro de Investigación y Docencia Económica y Conferencia Mexicana de Acceso a la Información Pública, 2014, Métrica de la transparencia 2014, Ciudad de México, CIDE-COMAIP.

Centro de Investigación y Docencia Económica y Conferencia Mexicana de Acceso a la Información Pública, 2010, Métrica de la transparencia 2010, Ciudad de México, CIDE-COMAIP.

Coleman, John J., 1999, "Unified Government, Divided Government, and Party Responsiveness", The American Political Science Review, vol. 93, núm. 4.

Edwards, George C. et al., 1997, "The Legislative Impact of Divided Government”, American Journal of Political Science, vol. 41, núm. 2.

Elizondo, Carlos, 2012, Con dinero y sin dinero... Nuestro ineficaz, precario e injusto equilibrio fiscal, México, Debate.

Giraudy, Agustina, 2015, Democrats and Autocrats. Pathways of Subnational Undemocratic Regime Continuity within Democratic Countries, Oxford, Oxford University Press.

Gobierno del Estado de Chinuahua, 2010, Plan Estatal de Desarrollo, disponible en: http://www.chihuahua.gob.mx/atach2/contraloria/uploads/ Plan\%20Estatal\%20de\%20Desarrollo\%202010-2016\%20Final.pdf.

Gobierno del Estado de Veracruz, 2011, Plan Veracruzano de Desarrollo, disponible en: http://www.veracruz.gob.mx/programadegobierno/plan-vera cruzano-de-desarrollo-2011-2016/.

Hartmann, Sebastian, 2015, Partisan Policy-Making in Western Europe. How Ideology Influences the Content of Government Policies, Springer, Mannheim.

Herwartz, Helmut y Theilen, Bernd, 2014, "Partisan influence on social spending under market integration, fiscal pressure and institutional change", European Journal of Political Economy, vol. 34.

Hibbs, Douglas A., 1977, "Political Parties and Macroeconomic Policy", The American Political Science Review, vol. 71, núm. 4.

Howell, William et al., 2000, "Divided Government and the Productivity of Congress, 1945-1994”, Legislative Studies Quarterly, vol. 25, núm. 2.

IbARRA, Jorge, 2013, "Entorno político y dependencia financiera de los estados mexicanos", Gestión y Política Pública, vol. 22, núm. 3. 
Esta revista forma parte del acervo de la Biblioteca Jurídica Virtual del Instituto de Investigaciones Jurídicas de la UNAM

Índice Nacional de los Órganos Garantes del Derecho de Acceso a LA Información, 2016, Resultados 2016, disponible en: https://inogdai. org/\#!/mapa? indice $=2016$ Eestado=nacional $)$.

Índice Nacional de los Órganos Garantes del Derecho de Acceso a LA Información, 2014, Resultados 2014, disponible en: https://inogdai. org/\#!/mapa? indice $=2014$ Eestado=nacional .

Ingram, Matthew C., 2012 "Elections, Ideology, or Opposition? Assesing Competing Explanations of Judicial Spending in the Mexican States", The Journal of Law, Economics, E Organization, vol. 29, núm. 1.

Kelly, Sean Q., 1993, "Divided We Govern? A Reassessment", Polity, vol. 25, núm. 3.

Kernell, Samuel, 1991, "Facing an Opposition Congress: The President's Strategic Circumstance”, en Cox, Gary y Kennell, Samuel (eds.), The Politics of Divided Government, Boulder, Westview Press.

KiEwiet, Roderick y McCubbins, Mathew D., 1985a, "Congressional Appropriations and the Electoral Connection", The Journal of Politics, vol. 47, núm. 1.

KiEwiet, Roderick y McCubbins, Mathew D., 1985b, "Appropiations Decisions as a Bilateral Bargaining Game Between President and Congress", Legislative Studies Quarterly, vol. 10, núm. 2.

Kittel, Bernhard y Obinger, Herbert, 2003, "Political parties, institutions, and the dynamics of social expenditure in times of austerity", Journal of European Public Policy, vol. 10, núm. 1.

LinZ, Juan José, 1998, "Democracia presidencial o parlamentaria. ¿Qué diferencias implican?”, en Linz, Juan José y ValenZuela, Arturo (eds.), La crisis del presidencialismo. El caso de América Latina, Madrid, Alianza.

Loftis, Matt y Mortensen, Petter, 2017, "A new approach to the study of partisan effects on social policy", Journal of European Public Policy, vol. 24, núm. 6.

Mainwaring, Scott y Shugart, Matthew, 2002, "Presidencialismo y sistema de partidos en América Latina”, en Mainwaring, Scott y Shugart, Matthew (eds.), Presidencialismo y democracia en América Latina, Buenos Aires, Paidós.

Mayhew, David, 1991, Divided We Govern. Party Control, Lawmaking and Investigations, 1946-1990, New Haven, Yale University Press.

McCubbins, Matthew, 1991, "Government on Lay-Away: Federal Spending and Deficits Under Divided Party Control”, en Cox, Gary y Kernell, 
Samuel (eds.), The Politics of Divided Government, Boulder, Westview Press.

McDonald, Michael et al., 2007, "Cross-temporal and cross-national comparisons of party left-right positions", Electoral Studies, vol. 26.

Montemayor, Óscar, 2003, "Determinantes del endeudamiento subnacional. Un estudio para los estados y municipios de México, 1993-1999”, Ensayos, vol. 22, núm. 1.

Morales, Alberto, 2016, "Estados ahorcan a la transparencia", El Universal, 5 de octubre, disponible en: https:/wwww.eluniversal.com.mx/articulo/ nacion/politica/2016/10/5/estados-ahorcan-la-transparencia).

Nacif, Jenny, 2005, Para entender el Poder Legislativo de los Estados Unidos Mexicanos, México, Nostra.

Potrafke, Niklas, 2011, "Does government ideology influence budget composition? Empirical evidence from OECD countries", Economics of Governance, vol. 12, núm. 2.

Russo, Federico y Verzichelli, Luca, 2016, "Government ideology and party priorities: the determinants of public spending changes in Italy", Italian Political Science Review, vol. 26.

Schmidt, Manfred, 2010, "Parties", en Castles, Francis et al. (eds.), The Oxford Handbook of the Welfare State, Oxford, Oxford University Press.

Sснмітт, Carina, 2016, "Panel data analysis and partisan variables: how periodization influence partisan effects", Journal of European Public Policy, vol. 23, núm. 10.

Sundquist, James, 1988, "Needed: A Political Theory of the New Era of Coalition Government in the United States", Political Science Quarterly, vol. 103, núm. 4.

Urrutia, Alonso, 2016, "Organismos garantes de transparencia enfrentan insuficiencia de recursos", La Jornada, disponible en: https://www.jornada. com.mx/2016/01/25/politica/016n1pol.

Vassallo, Francesca y Wilcox, Clyde, 2006, "Party as a carrier of ideas", en Katz, Richard у Свотту, William J. (eds.), Handbook of Party Politics, Londres, Sage.

Velázquez, César, 2006, "Determinantes del gasto estatal en México", Gestión y Política Pública, vol. 15, núm. 1. 